\title{
Elastic-plastic simulation of plate with a blunt slit subjected to uni-axial tension
}

\author{
S. Ohtaki ${ }^{1}$, S. Kobayashi ${ }^{2} \&$ T. Yamamoto ${ }^{3}$ \\ ${ }^{1}$ Department of Mechanical Systems Engineering, \\ Hokkaido Institute of Technology, Japan \\ ${ }^{2}$ Actis Co., Japan \\ ${ }^{3}$ J-tec Co., Japan
}

\begin{abstract}
By the progress of a computer, the elastic-plastic simulation becomes easier under the mathematical assumption. However, results of the numerical analysis of the elastic-plastic problem depend on the assumption of yield conditions, definition of yield points and approximation of the stress-strain diagram of the material and other factors. The best way to testify the precision is to compare the numerical results with the experimental ones. In this paper, the authors study a numerical simulation of the elastic-plastic problem under plane stress by the finite element method. Calculation is executed by using FEM software LUSAS. The plastic theory is based on the strain incremental one, and Prandtl-Reuss equations are used. As a yield condition, von Mises' one is adopted. The definition of the yield stress is determined by the proportional limit. Three approximations of stress-strain diagrams are selected. To verify the numerical results, an experiment is conducted under the same condition by the photoelastic coating method. However, it is not easy to evaluate the stress distributions from the isochromatic fringes. A numerical example of thin plate with a blunt slit subjected to uni-axial tension is presented. Stress distributions in the minimum cross-section and stress contours are presented and examined. Keywords: structural analysis, finite element method, elastic-plastic problem, stress concentration.
\end{abstract}




\section{Introduction}

Machines and structures are usually composed of various kinds of metals. Stress concentration due to the existence of cracks or notches in metals causes fracture or failure of the structures. Therefore, it is important to investigate the elasticplastic behavior of metals. The finite element method is one of the most effective tools to deal with the numerical simulation of elastic-plastic problem.

Various mathematical methods in the theory of plasticity have been proposed by many researchers $[1-3]$. Also, Ellyin and $\mathrm{Wu}$ analyzed the stationary crack simulation under the cyclic loading containing the effect by the over- loading cycle using their constitutive equations [4].

In this paper, simulation of elastic-plastic problem is studied. To evaluate the validity of the numerical simulation of the elastic-plastic problem by the finite element method, it is desirable to compare the result with an experimental one by the photoelastic coating method (PCM) under the same dimensions and conditions [5,6]. Important factors in the elastic-plastic calculation are the definition of the yield points and the assumption of stress-strain curves [7]. For example, simple prediction of a stress-strain diagram is an approximation with two bi-linear lines, taking the work-hardening ratio constant value during the plastic deformation. There are several ways of the definition of yield points. Numerical simulations are conducted for three cases subjected to the tensile load. The software called LUSAS is used for the numerical calculation so as to consider the convenience and visualization [8]. The incremental step-by-step calculation is adopted in the convergent process of Newton-Raphson method. Numerical example is presented for a rectangular thin plate with a blunt slit subjected to uni-axial tensile load. Stresses and strains in the minimum crosssection and contour lines are shown. The contour lines called quasi-isochromatic fringes by the finite element model are compared with isochromatic ones obtained by the PCM experiment. And the reinforcing effect of the PCM is also simulated by using three-dimensional solid elements.

\section{Elastic-plastic theory}

\subsection{Yield function}

Considering the second and the third invariants of the deviatoric stress tensor $J_{2}^{\prime}$ and $J_{3}^{\prime}$, the yield condition $\mathrm{f}$ for the isotropic materials is written as the next equation.

$$
f=F\left(J_{2}^{\prime}, J_{3}^{\prime}\right)
$$

Where those two invariants can be expressed using the tensor notation as,

$$
\begin{aligned}
& J_{2}^{\prime}=\sigma_{i j}^{\prime} \sigma_{i j}^{\prime} / 2 \\
& J_{3}^{\prime}=\sigma_{i j}^{\prime} \sigma_{i j}^{\prime} \sigma_{i j}^{\prime} / 3
\end{aligned}
$$

Assuming that the third invariants $J_{3}^{\prime}$ does not affect the yielding behavior, the von Mises yield condition is adopted and it is given as, 


$$
\begin{aligned}
f & =6 j_{2}^{\prime}-2 \bar{\sigma} \\
& =\left(\sigma_{x}-\sigma_{y}\right)^{2}+\left(\sigma_{y}-\sigma_{z}\right)^{2}+\left(\sigma_{z}-\sigma_{x}\right)^{2}+6\left(\tau_{y z}^{2}+\tau_{z y}^{2}+\tau_{x y}^{2}\right)-2 \sigma_{Y}^{2}
\end{aligned}
$$

Under the plane stress, the yield surface is depicted with an elliptical form.

\subsection{Constitutive equations}

The relations between stress and strain increments under the plane stress are written as

In the elastic region

where

$$
\{d \sigma\}=\left[D^{e}\right]\{\varepsilon\}
$$

$$
\begin{gathered}
\{d \sigma\}=\left\{\begin{array}{llll}
\sigma_{x} & \sigma_{y} & \tau_{x y} & \}^{T}
\end{array}\right. \\
\{d \varepsilon\}=\left\{\begin{array}{cccc}
\varepsilon_{x} & \varepsilon_{y} & \gamma_{x y} & \}^{T}
\end{array}\right. \\
{\left[D^{e}\right]=\frac{E}{1-v^{2}}\left|\begin{array}{ccc}
1 & v & 0 \\
v & 1 & \frac{1-v}{2}
\end{array}\right|}
\end{gathered}
$$

In the plastic region

$$
\{d \sigma\}=\left[D^{p}\right]\{\varepsilon\}
$$

where

$$
\left[D^{P}\right]=\left[\begin{array}{ccc}
\frac{E}{1-v^{2}}-\frac{S_{1}^{2}}{S} & \frac{v E}{1-v^{2}}-\frac{S_{1} S_{2}}{S} & -\frac{S_{1} S_{6}}{S} \\
& \frac{E}{1-v^{2}}-\frac{S_{2}^{2}}{S} & -\frac{S_{2} S_{6}}{S} \\
S Y M . & \frac{E}{2(1+v)}-\frac{S_{6}^{2}}{S}
\end{array}\right]
$$

where

$$
\begin{gathered}
S=\frac{4}{9} \bar{\sigma}^{2} H^{\prime}+S_{1} \sigma_{x}^{\prime}+S_{2} \sigma_{y}^{\prime}+2 S_{6} \tau_{x y} \\
S_{1}=\frac{E}{1-v^{2}}\left(\sigma_{x}^{\prime}+v \sigma_{y}^{\prime}\right) \\
S_{2}=\frac{E}{1-v^{2}}\left(\sigma_{y}^{\prime}+v \sigma_{x}^{\prime}\right) \\
S_{6}=\frac{E}{1+v} \tau_{x y}
\end{gathered}
$$

where, E, $v, H^{\prime}$ and $\sigma_{i}^{\prime}$ are Young's modulus, Poisson's ratio, work-hardening ratio and deviatoric stresses, respectively. 


\section{Finite element calculation}

\subsection{Configuration}

Numerical example of a thin plate with a blunt slit under the plane stress has been calculated by the finite element method. Originally, the shape of the model is the same as the specimen conducted by the photoelastic coating method. The material used for the PCM experiment is made of aluminum alloy (A5052PH34) and the mechanical properties are shown in Table 1. Yield surfaces under the plane stress are depicted in Fig.1. The shape of the specimen and its dimensions are shown in Fig. 2. Without taking into account the geometrical symmetric condition, whole specimen is divided into the finite element model as shown in Fig. 3 using auto-mesh function. The total number of elements is 16176, and that of nodal points is 14680 .

Table 1: Mechanical properties and yield stress.

\begin{tabular}{|c|c|c|c|}
\hline & $\begin{array}{c}\text { YOUNG'S } \\
\text { MODULUS } \\
\text { GPa }\end{array}$ & $\begin{array}{c}\text { POISSON'S } \\
\text { RATIO }\end{array}$ & $\begin{array}{c}\text { YIELD } \\
\text { STRESS } \\
\text { MPa }\end{array}$ \\
\hline $\mathrm{H} 34$ & 72.7 & 0.29 & 120 \\
\hline
\end{tabular}

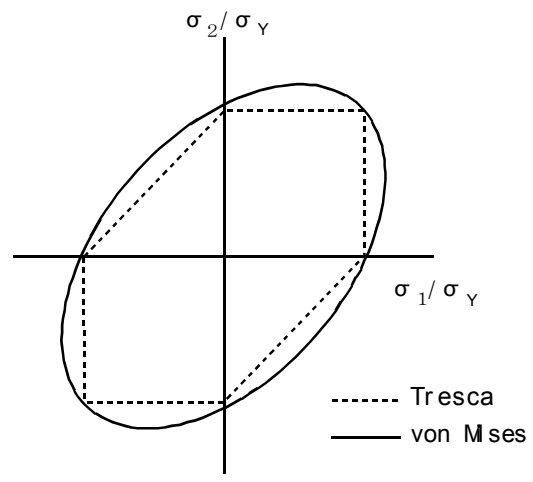

Figure 1: $\quad$ von Mises and Tresca yield surfaces.

\subsection{Yield points and stress-strain diagram}

There are many definitions of yield points. We used two kinds of definitions. One is the proportional limit and the other is the intersection between two straight lines. In the elastic-plastic calculation, there are some approximate ways to predict the stress-strain diagram. One of the simplest ways is to approximate with bi-linear lines. The precise prediction of the stress-strain diagram is to use multi-linear lines to express the gradual change of work-hardening ratio $\mathrm{H}^{\prime}$. In the calculation of the plastic region, we deal with three cases. In the Case 1, the 
yield point is the proportional limit and work-hardening ratio is taken as a constant. In the Case 2, the yield point is defined with intersection of the two extrapolated lines and $\mathrm{H}^{\prime}$ is constant. In the Case 3, stress-strain curve is approximated with twelve straight lines. Stress-strain diagrams for three cases are shown in Fig. 4.
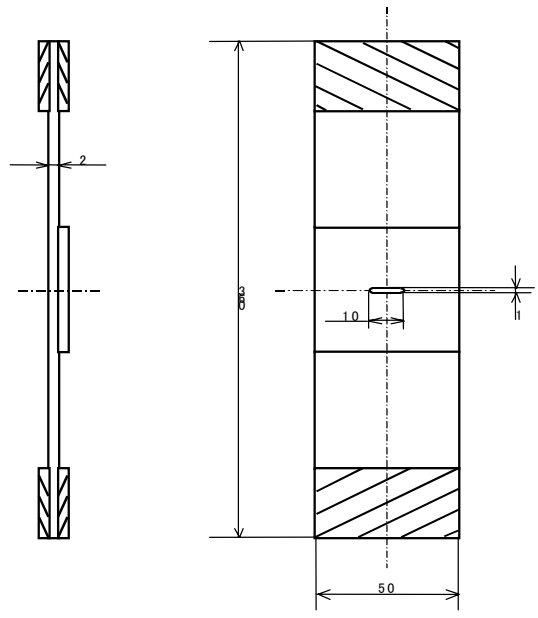

Figure 2: Rectangular specimen with a blunt slit.

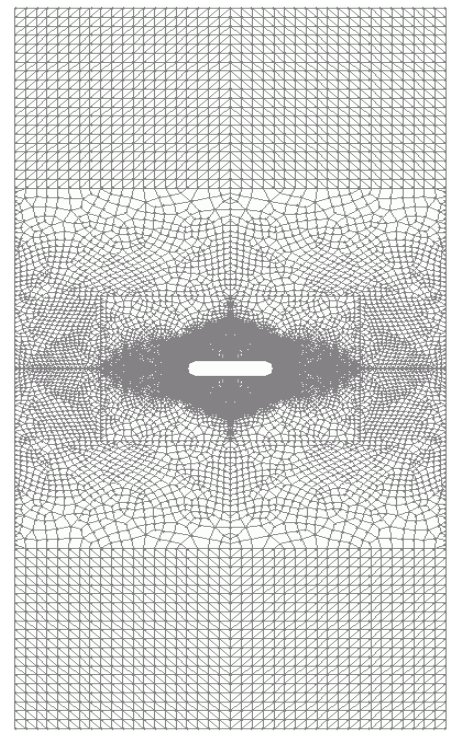

16176 elements, 14680 nodes

Figure 3: $\quad$ Finite element model. 


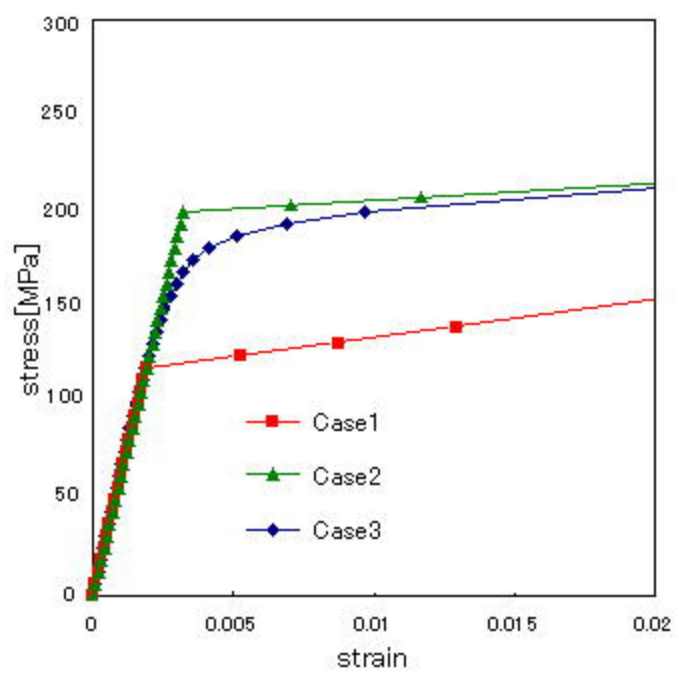

Figure 4: $\quad$ Stress-strain diagrams of the aluminum alloy.

\section{Numerical results}

The stress component $\sigma_{y}$ of the tensile direction in the minimum cross-section for the Case 1 is presented in Fig. 5 for several loading steps. Similarly, $\sigma_{y}$ for the Case 2 is shown in Fig. 6. In the Cases 1 and 2, as the work-hardening ratios $\mathrm{H}^{\prime}$ are assumed to be constant, the maximum stresses near the tip of the slit are constant too.
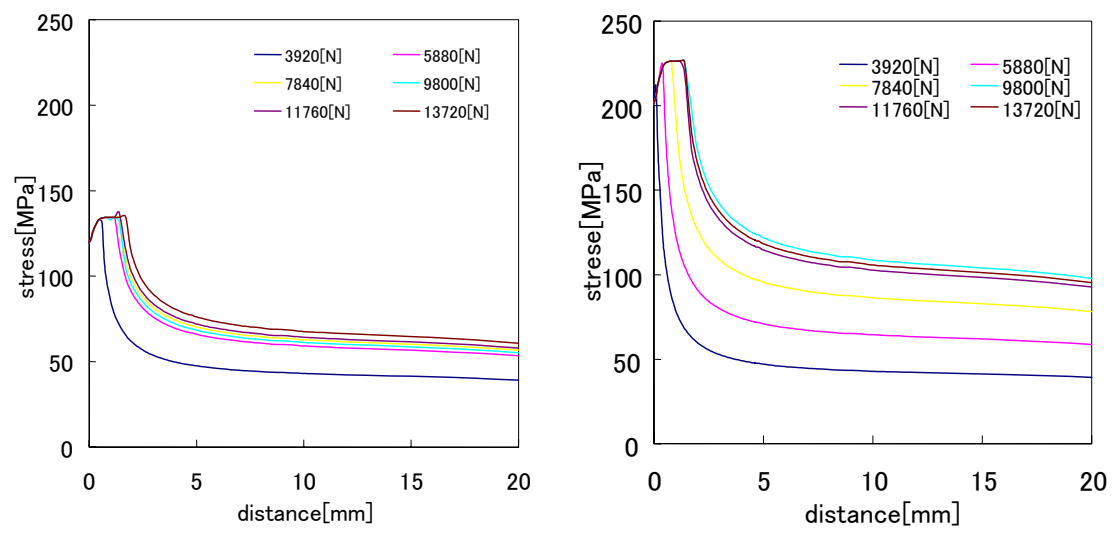

Figure 5: $\sigma_{y}$ in the x-axis (Case 1). Figure 6: $\sigma_{y}$ in the x-axis (Case 2). 


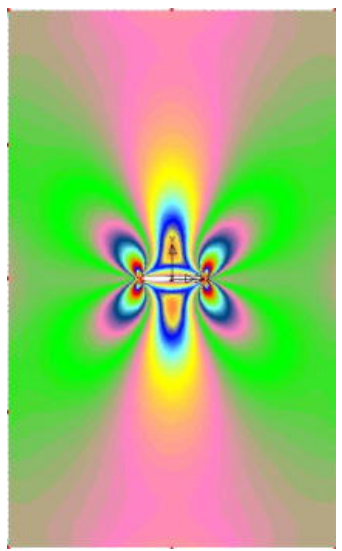

Figure 7: Quasi-isochromatic fringe Figure 8: Isochromatic fringe by by $\operatorname{FEM}\left(\mathrm{P}_{\mathrm{y}}=11760 \mathrm{~N}\right)$.

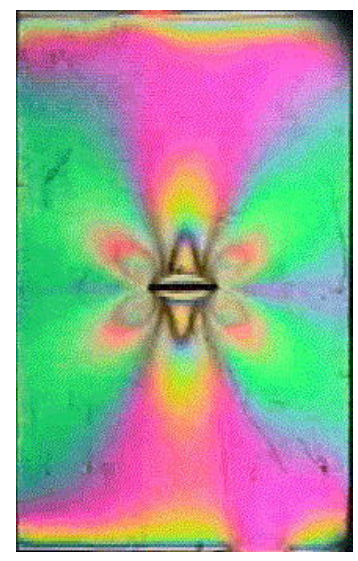

$\operatorname{PCM}\left(\mathrm{P}_{\mathrm{y}}=11760 \mathrm{~N}\right)$.

The difference of definition of the yield points makes significant difference in the magnitude of stress distribution. In the case 3, the increase of the change of $\sigma_{y}$ in the plastic range is evident. From this fact, the numerical calculation had conducted using the stress-strain diagram of the case 3 .

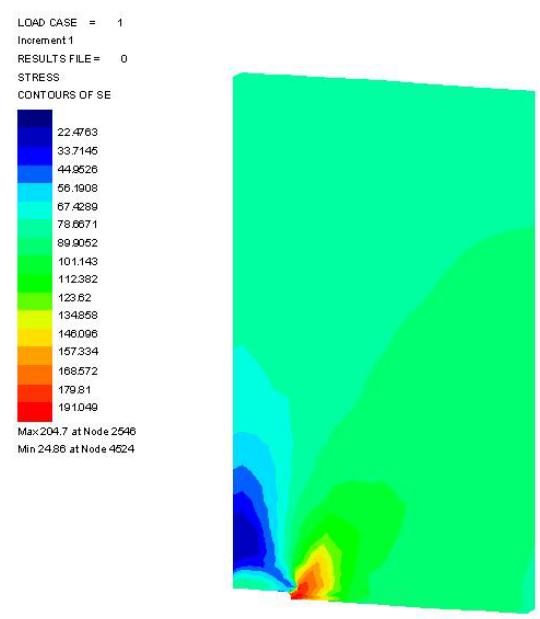

Figure 9: Equivalent stress $\bar{\sigma}$ without coating in case of $\mathrm{P}_{\mathrm{y}}=8829 \mathrm{~N}$ (Von Mises). 


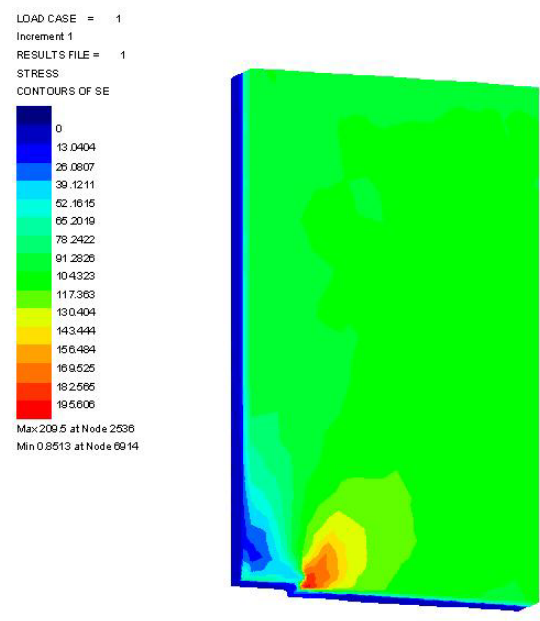

Figure 10: Equivalent stress $\bar{\sigma}$ with coating in case of $\mathrm{P}_{\mathrm{y}}=8829 \mathrm{~N}$ (Von Mises).

In the calculation with LUSAS, the von Mises yield criterion is adopted. Therefore, we can easily obtain the contour lines of equivalent stress. To compare the results by PCM experiment, Tresca's quasi-isochromatic lines that are proportional to the difference of two principal stresses are calculated and shown in Fig. 7 for the case of $\mathrm{P}_{\mathrm{y}}=11760 \mathrm{~N}$. The isochromatic fringes conducted by the PCM experiment are presented in Fig. 8 for the same loading step. The tendency of these results is roughly similar to each other, though quantitative analysis is not adequate yet due to the limitation of the maximum number of color values of the software. Thus, without complicated calculation of stress and strains of PCM experimental procedure, the validity of the results by the finite element can be assured by the comparison of these two Figs. 7 and 8 .

It is known that if the stiffness of the polymer coating which is adhered on the surface of the specimen in the PCM is larger compared with the surface metal, the reinforcing effect is not negligible. We have calculated the reinforcing effect with three-dimensional solid elements taking account of the stiffness in the thickness direction of the aluminum plate and polymer. The contour lines of equivalent stress $\bar{\sigma}$ without coating in case of $\mathrm{P}_{\mathrm{y}}=8829 \mathrm{~N}$ are shown in Fig. 9. Similarly, contour lines of $\bar{\sigma}$ with coating are shown in Fig.10. However, from these figures, the comparison of the quantities of the equivalent stress is not clear because of the difference of the color scale. By plotting the equivalent stress in the minimum cross-section, the reinforcing effect is calculated as 6 percent.

\section{Conclusion}

In this paper, the finite element simulation has been conducted for the thin plate with a blunt slit subjected to uni-axial tension. Three cases had been calculated 
with different definition of yield points and the approximation of stress-strain diagrams in the proportional tensile load. The stress distribution in the minimum cross-section and stress contour lines are obtained.

(1) The definition of yield point affects the magnitude of the distribution of stress state in plastic range greatly. The best approximation of stressstrain diagram is to use the proportional limit as the yield point and multi-linear work-hardening ratios of case 3 .

(2) The evolution of elastic-plastic boundaries is roughly similar to those obtained by the photoelastic coating experiment. However, quantitative analysis is not adequate in the present method.

(3) The reinforcing effect of the polymer of the PCM experiment is simulated by three dimensional solid elements. It is found that the reinforced effect is about 6 percent which coincide with theoretical value.

\section{References}

[1] Marcal, P.V. and King, I.P., Elastic-plastic analysis of two-dimensional stress by the finite element method, International Journal of Mechanical Science, 9-3, pp. 143-155, 1967.

[2] Zienkiwitcz, O.C., The finite element method, Third Ed. McGraw-Hill, 1977.

[3] Owen, D.R.J. and Hinton, E., Finite element in Plasticity (Theory and Practice), Pineridge Press Limited, Swansea, UK, 1980.

[4] Ellyin, F. and Wu, J., Elastic-plastic analysis of a stationary crack under cyclic loading and effect of over load, International Journal of Fracture, 56, pp. 189-208, 1992.

[5] Zandman, F. et al., Photoelastic Coating, ISU Press \& SEM, 1977.

[6] Theocaris, P.S., Experimental Mechanics, 3-9, 1963.

[7] Ikegami, K., A Historical Perspective of the Experimental Study of Subsequent Yield Surface for Metal-Part 1.2, British Industrial \& Scientific International Translation Service, pp.1-33, 1976.

[8] LUSAS theory manual Version 13, FEA Ltd., UK, 2000. 